\title{
Printed polymers for poorly plants
}

Sci. Adv. 5, eaaw0463 (2019)

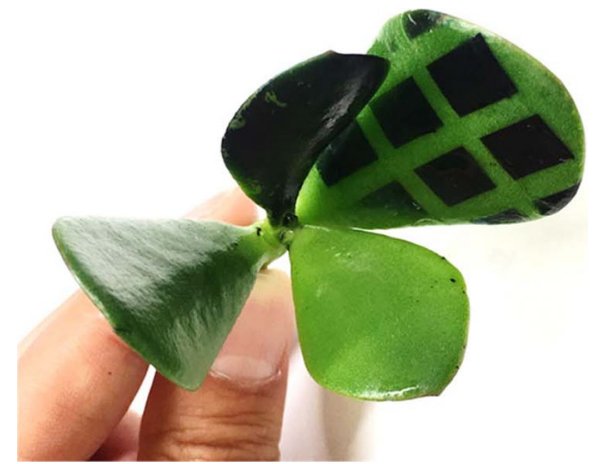

Credit: AAAS under a Creative Commons license CC BY-NC 4.0

New classes of flexible medical sensors that are applied directly onto living tissue require attachable electrodes for monitoring. However, many organic surfaces are covered by microscopic features that prevent contacts from sticking effectively. These features, which often perform roles vital to an organism's health, can also be adversely affected by the presence of the contacts. Trisha Andrew and colleagues at the University of Massachusetts Amherst have now created robust and bio-friendly polymer electrodes that can be deposited directly onto living plants.

To create these contacts, a technique known as vapour printing was used.
In particular, the plant was sealed in a chamber and a mixture of vapourized monomer and oxidant introduced. The oxidant induces a polymerization reaction, which grows a film over any exposed surface. The resultant coatings were found to conform to any plant surface tested, regardless of its microscopic complexity. Additionally, the contacts remained adhered to the surface as the plants grew and caused no observable ill-effects to the plant's health.

The polymer layers were used to make conductive pads for bioimpedance spectroscopy, which can measure changes in tissue resistivity. Such measurements have been used to monitor plant health, but previously required piercing the sample with metal needles. Andrew and colleagues showed that their bioimpedance measurements, taken using the vapourgrown contacts, can successfully detect leaf tissue damage caused by drought and other factors. The robustness of the contacts, combined with the minimal invasiveness of the approach, also meant that tissue health could be monitored on-demand over the lifetime of the plant.

Matthew Parker

Published online: 15 April 2019

https://doi.org/10.1038/s41928-019-0239-9 